\title{
Advancing Social Media and Mobile Technologies in Healthcare Education
}

\author{
Elizabeth Cummings ${ }^{1, *}$ (i) and Carey Mather ${ }^{2}$ \\ 1 School of Health Sciences, Faculty of Health, University of Tasmania, Hobart 7001, Australia \\ 2 School of Health Sciences, Faculty of Health, University of Tasmania, Launceston 7250, Australia; \\ Carey.Mather@utas.edu.au \\ * Correspondence: Elizabeth.Cummings@utas.edu.au
}

Academic Editor: Antony Bryant

Received: 15 September 2017; Accepted: 27 September 2017; Published: 28 September 2017

Keywords: mobile technologies; social media; healthcare; education

Social media and mobile technologies are important new tools in healthcare education, both to assist healthcare professionals learn and maintain their craft, and for the education of patients and families. Social media, particularly linked with mobile technologies, are being used to support healthcare professionals in higher education and maintaining professional development in the workplace. They are also becoming important as tools for educating patients and their families in relation to healthcare needs. This special issue sought submissions offering research results and case studies that advance the methodologies aimed at the application of social media or mobile technologies for healthcare education.

Skiba identifies and reviews criteria and instruments that can be used to evaluate mobile apps and social media. She reviews current literature to facilitate healthcare professionals' assessment of mobile apps and social media tools that may be pertinent to their patient population. The review highlights strategies that a healthcare system can incorporate to provide guidance for recommended mobile apps and social media tools for patients, families, and caregivers.

Procter, Brixley, Todhunter, and Honey consider the opportunities advances in social media offer for developing the understanding of different countries and cultures without needing to travel. Despite nursing's global presence, students have limited knowledge of the health and social care needs and provision outside their local environment. The paper reports on three countries, New Zealand, the United Kingdom, and the United States of America, and their collaborative efforts to provide senior student nurses with a communication channel to explore public health issues in each country. Using a closed Facebook ${ }^{\mathrm{TM}}$ page, third-year undergraduate adult nursing students were invited to take part in a three-month pilot study to test the feasibility of virtual collaboration through exchanging public health issues. The research demonstrated that social media offers students an opportunity to engage meaningfully in global exchanges in a virtual form.

The paper by Mather and Cummings explores nurse supervisors' use of mobile technology for informal learning and continuing professional development, both for their own professional practice and in their role in modelling digital knowledge transfer, by facilitating the learning and teaching of nursing students in the workplace. It emphasises the necessity of implementing mobile learning at the point of care to enable digital knowledge transfer, augment informal learning for students and patients, and support continuing professional development opportunities for all staff.

The imperative for embedding digital professionalism into undergraduate health profession courses and augmenting learning and teaching with mobile technology and social media on and off campus is the focus of the paper by Mather, Douglas, and O'Brien. Their paper explores whether patterns of social media use for personal or informal learning by Australian undergraduate health 
profession students changed over time. Using a validated online survey administered at two points (2013 and 2016), a comparative analysis of the two cohorts was undertaken, demonstrating an increase in social media acceptance and penetration. The authors argue that this creates opportunities to integrate social media into the health profession education curriculum.

Understanding student nurses' use of social media in relation to their learning is the focus of the paper by Honey and Raphael. Surveying undergraduate nursing students at a New Zealand university in 2015, they identified that while almost all students use social media outside their studies, less than two thirds of the study sample use social networking sites on a daily basis in relation to their study. The most common digital option used to communicate and work with fellow students was online groups and document sharing sites. These results concur with those of Mather, Douglas, and O'Brien, and further the evidence for educators to incorporate social media into teaching and learning activities, including its safe and ethical use.

Finally, Fan, Radford, and Fabian report the results of the development and implementation of a social media technology supported website-digiMe at the School of Medicine of an Australian university. digiMe provided medical students with the opportunity to connect with other medical learners and health professionals. digiMe demonstrated its potential in raising awareness of web and mobile apps useful for enhancing connectivity, although it needs to be introduced to students in earlier years of their medical education to achieve a higher impact on their learning.

Each of these articles focuses on different aspects of the use of mobile technologies and social media for healthcare education. The papers provide a sample of the ongoing commitment by educators to advance the use of technology within healthcare and in higher education by examining aspects of integration. These studies inform the literature about this rapidly evolving topic. Others can learn from the findings and further progress the use of mobile technologies and social media in healthcare education. 